This is a postprint version of the following published document:

Pozuelo, J., Riande, E., Saiz, E. \& Compañ, V. (2006): Molecular Dynamics Simulations of Proton Conduction in Sulfonated Poly(phenyl sulfone)s. Macromolecules, 39 (25), pp.: 8862-8866.

"This document is the Accepted Manuscript version of a Published Work that appeared in final form in Macromolecules, (c) 2006 American

Chemical Society after peer review and technical editing by the publisher.

To access the final edited and published work see http://pubs.acs.org/doi/abs/10.1021/ma062070h, or see DOI: $\underline{10.1021 / \mathrm{ma} 062070 \mathrm{~h}} "$ 


\title{
Molecular Dynamics Simulations of Proton Conduction in Sulfonated Poly(phenyl sulfone)s
}

\author{
Javier Pozuelo, ${ }^{\dagger}$ Evaristo Riande, ${ }^{*, *}$ Enrique Saiz, ${ }^{\S}$ and Vicente Compañ ${ }^{\perp}$ \\ Departamento de Ciencia e Ingeniería de Materiales e Ingeniería Química, Universidad Carlos III, \\ Leganés, Madrid, Spain; Instituto de Ciencia y Tecnología de Polimeros (CSIC), 28006 Madrid, Spain; \\ Departamento de Química Física, Universidad de Alcalá, Alcalá de Henares, Madrid, Spain; and \\ Deparatamento de Termodinámica Aplicada, ETSII, Universidad Politécnica de Valencia, \\ Valencia, Spain
}

\begin{abstract}
Full molecular dynamics was used to simulate separately the diffusion of naked protons and $\mathrm{H}_{3} \mathrm{O}^{+}$hydrated protons across sulfonated poly(phenyl sulfone)s. Simulations were carried out for wet membranes with the following characteristics: ion-exchange capacity, 1.8 mequiv/g of dry membrane; water uptake, $10-30 \%$; temperature range, $300-360 \mathrm{~K}$. The diffusion coefficient of naked protons is nearly 1 order of magnitude higher than that of the hydrated protons for the membranes with the lower water uptake (10\%). For the membranes with higher water uptake the ratio between the diffusion coefficients of the two particles reduces to about half an order of magnitude. The conductivity of the naked protons increases from $21.4 \times 10^{-3}$ to $52.5 \times 10^{-3} \mathrm{~S} / \mathrm{cm}$ when the water uptake increases from $10 \%$ to

$30 \%$. For hydrated protons the conductivity increases from $1.54 \times 10^{-3}$ to $7.57 \times 10^{-3} \mathrm{~S} / \mathrm{cm}$. The conductivities obtained through simulations carried out at $300 \mathrm{~K}$ for the hydrated proton across membranes with water uptake $18 \%$ and $30 \%$ are roughly similar to those experimentally measured for a membrane with ion exchange capacity $=1.8$ mequiv/g and water uptake $=24.3 \%$. Simulated conductivities of both naked protons and hydrated protons follow Arrhenius behavior.
\end{abstract}

\section{Introduction}

After the synthesis of ion-exchange resins in the 1930 s, the dispersion of finely powdered resins into a binding polymer gave rise to the development of ion-exchange membranes. The first synthetic ion-exchange membranes were reported in the $1950 \mathrm{~s} .{ }^{1,2}$ Since then, a great variety of ion-exchange membranes have been prepared as a result of the ever-increasing use of these materials in separation processes, synthesis of high-purity substances, batteries, fuel cells, etc. Good performance ionexchange membranes for a variety of applications should exhibit high ionic permselectivity, high ionic conductance, low permeability to free diffusion of electrolytes, and low electroosmosis as well as chemical stability, mechanical resistance, flexibility, and good dimensional stability in usually unfriendly working conditions. $^{3-5}$ Nevertheless, some of these properties are incompatible. For example, high mechanical resistance requires thick membranes, which is incompatible with high conductance. High ion-exchange capacity increases permeselectivity and conductance, but it may be a negative factor for both dimensional stability and mechanical resistance. From a practical point of view, good performance membranes must exhibit a suitable balance of incompatible properties.

The synthesis of cation-exchange membranes that combine low protonic resistance and good mechanical properties in adverse operating conditions is nowadays a flourishing field of research. ${ }^{6}$ Although perfluorocarbon polymers conveniently sulfonated are the best materials that meet these requirements, their still high cost and negative environmental effects make necessary to look for alternative materials. In principle, polymers with high chemical stability and good mechanical properties, such as polysulfones, polyimides, poly(ether ketone)s, etc., are suitable materials to prepare acidic membranes. ${ }^{6}$

\footnotetext{
${ }^{\dagger}$ Universidad Carlos III

‡ Instituto de Ciencia y Tecnología de Polímeros

$\S$ Universidad de Alcalá

${ }^{\perp}$ Universidad Politécnica de Valencia.
}

Theoretical studies of polymer electrolytes through molecular dynamics are important for the design of good performance conducting membranes. Realistic models of ionic salts for poly(ethylene oxide)-inorganic salt (for example $\mathrm{NaI}$ ) suggest that the ions in the conductive process slide along a polymer chain by breaking bonds between ions and polymer segments and renewing these bonds with other segments of the same or another polymer. ${ }^{7-9}$ Accordingly, there is a strong coupling between ion motion and segmental relaxation in such a way that an understanding of ionic conduction in polyelectrolytes requires a detailed knowledge of segmental relaxations in these systems. Models have been reported that depict particle motion as hopping between neighboring sites or ion coordinating sites on a two-dimensional lattice. Chain motions are simulated by periodically redistributing or "repercolating" the hopping paths at relaxation times $\tau_{\mathrm{r}}$ in such a way that the model reproduces the dependence of ion transport on polymer motion. ${ }^{10}$

Though proton diffusion in cation-exchange membranes is a not well-understood phenomenon, this process presumably involves dissociation of the proton from the acidic site, subsequent transfer of the proton to the aqueous medium, screening by water of the hydrated proton from the conjugated base or fixed anion, and finally diffusion of the proton in the confined water within the polymer matrix. ${ }^{11}$ Therefore, the dependence of proton mobility on water content is quite critical and demands effective cell and stack design to maintain a high level of water through the thickness of the membrane for the complete range of dynamic operation. It is worth noting that both evaporation and electroosmotic drag processes severely diminish membranes humidification at typical working temperatures of fuel cells, causing a significant increase of the protonic resistance.

In this work, the trajectories of naked protons and hydrated protons $\left(\mathrm{H}_{3} \mathrm{O}^{+}\right)$in sulfonated poly(phenyl sulfone) membranes are simulated using full molecular dynamics techniques at different temperatures lying in the range $25-60{ }^{\circ} \mathrm{C}$. Once the 
trajectories reach Einstein behavior, the diffusion coefficients of the mobile ions are determined, from which the conductivities are obtained. The simulations were carried out for polysulfone cation-exchange membranes with different water content, keeping the ion-exchange capacity constant, and the results for the conductivity obtained were further compared with experimental results.

\section{Experimental Section}

Sulfonation of Polysulfones. The sulfonation of the commercial poly(phenyl sulfone) Radel was carried out by dropwise adding trimethylsilylchlorosulfonate to a chloroform solution of the polymer. During this step, trimethylsilylsulfonate moieties are bound to the phenyl groups of the polysulfone chains and hydrochloric acid is released. Then an excess of methanol was added to the reaction medium converting the trimethylsilylsulfonate moieties into sulfonic groups, releasing tetramethylsiloxane. ${ }^{12}$ The polymer was precipitated with methanol, dissolved in chloroform, and precipitated again with methanol, washed with water, and dried overnight in a vacuum at $60^{\circ} \mathrm{C}$.

Ion-Exchange Capacity. Cation-exchange membranes were obtained by casting from poly(phenyl sulfonated sulfone) solutions. The ion-exchange capacity of the membranes was obtained by soaking them, in the acidic form, in a sodium chloride solution (1 $\mathrm{M})$. The protons released in the ion-exchange reaction $\mathrm{R}-\mathrm{SO}_{3} \mathrm{H}$ $+\mathrm{Na}^{+} \rightarrow \mathrm{R}-\mathrm{SO}_{3} \mathrm{Na}+\mathrm{H}^{+}$were titrated with a sodium hydroxide solution, obtaining for the ion-exchange capacity the value of 1.8 mequiv/g of dry membrane.

Water Uptake. Dried membranes in the acidic form were weighed and further immersed in distilled water overnight. Then the membranes were gently pressed between filter paper and weighed again. The number of molecules of water per ion-exchange site was $24 \%$.

Protonic Resistance. In principle, ion-exchange membranes behave like a resistance $R_{0}$ that accounts for the protonic conductivity in series with an ideal $R_{\mathrm{p}}-C$ parallel circuit, where $R_{\mathrm{p}}$ represents the polarization electrode resistance and $C$ the capacity. The complex impedance $Z^{*}$ of the system is given by

$$
Z^{*}=R_{0}+\frac{R_{\mathrm{p}}}{1+j R_{\mathrm{p}} \omega C}
$$

Equation 1 leads to the following expressions for the real, $Z^{\prime}$, and loss, $Z^{\prime \prime}$, components of the complex impedance, $Z^{*}$

$$
\begin{aligned}
& Z^{\prime}=R_{0}+\frac{R_{\mathrm{p}}}{1+\left(\omega R_{\mathrm{p}} C\right)^{2}} \\
& Z^{\prime \prime}=-\frac{\omega R_{\mathrm{p}}^{2} C}{1+\left(\omega R_{\mathrm{p}}^{2} C\right)^{2}}
\end{aligned}
$$

Values of the complex impedance were obtained at $25^{\circ} \mathrm{C}$ in the frequency domain by placing the membrane between two gold electrodes of a broadband dielectric spectrometer Novocontrol. According to eqs 2 and 3, the modulus of the impedance $\left|Z^{*}\right|$ decreases with increasing frequency, reaching a plateau at a frequency at which the phase angle vanishes, and the angle phase $\phi\left(=\arctan Z^{\prime \prime} / Z^{\prime}\right)$ reaches a maximum $(\phi=0)$ at the plateau. The value of $\left|Z^{*}\right|$ at this frequency is equal to the protonic resistance, $R_{0}$. The value of $R_{0}$ leads to the protonic conductivity $\sigma$ using the following expression

$$
\sigma=\frac{l}{R_{0} A}
$$

where $A$ and $l$ are respectively the area and thickness of the membranes. The value of the conductivity of the membrane at 300 $\mathrm{K}$ was $7.2 \times 10^{-3} \mathrm{~S} / \mathrm{cm}$.
Mean-Square Displacements and Protonic Diffusion. The relationship between conductivity and ionic diffusion in cationexchange membranes can be obtained using Nernst-Planck ionic transport formalism. In a cation-exchange membrane partially or totally equilibrated with water, electric current under a unidirectional electric force field, $\mathrm{d} \psi / \mathrm{d} x$, is transported by counterions. The motion of the counterions is accelerated by driving force $\mathrm{d} \psi / \mathrm{d} x$ and delayed by the friction of the moving particles against the surroundings. In steady-state conditions

$$
c_{+} z_{+} F \frac{\mathrm{d} \psi}{\mathrm{d} x}+\chi_{+} u_{+}=0
$$

where $F$ is Faraday's constant and $c_{+}$and $z_{+}$are respectively the concentration of the counterion and its valence, whereas $\chi_{+}$and $u_{+}$are respectively the friction coefficient of the particles against the surroundings and their velocity in the direction of the force field. Taking into account the Einstein's relationship $\chi_{+}=R T / D_{+}$ where $D_{+}$is the diffusion coefficient of the counterion and $R T$ has the usual meaning, the flux of the counterions, $J_{+}$, across the cationexchange membrane is given by

$$
J_{+}=c_{+} u_{+}=-c_{+} \bar{u}_{+} \frac{\mathrm{d} \psi}{\mathrm{d} x}
$$

where the convention flow has been neglected. In this expression, $\bar{u}_{+}=F z_{+} D_{+} / R T$ is the ionic mobility of the counterion. Since the current density is $j=F z_{+} J_{+}$, the electrical conductance can be written as

$$
\sigma=-\frac{j}{\mathrm{~d} \psi / \mathrm{d} x}=F z_{+} c_{+} \bar{u}_{+}=\frac{c_{+}\left(F z_{+}\right)^{2} D_{+}}{R T}
$$

Equation 7 can be expressed in a more conventional way for simulation calculations by

$$
\sigma=\frac{N_{+} z_{+}{ }^{2} e^{2} D_{+}}{V k T}
$$

where $N_{+}$is the number counterions in the simulation box of volume $V, k$ is the Boltzmann constant, and $T$ is the absolute temperature. The conductance is often written as

$$
\sigma=\frac{N_{+} z_{+}{ }^{2} e^{2} D_{+}}{V k T H_{\mathrm{R}}}
$$

where $H_{\mathrm{R}}=D_{+} / D_{\sigma+}$ is the Haven ratio, a factor that compensates for ion correlations, and $D_{\sigma+}$ is the actual diffusive conductivity. In the simulations $H_{\mathrm{R}}$ was set to $1 .^{14}$

Diffusion coefficients $D_{+}$of counterions in cation-exchange membranes can be calculated by the Einstein diffusion equation:

$$
D_{+}=\frac{1}{6 N_{+}} \lim _{t \rightarrow \infty} \frac{\mathrm{d}}{\mathrm{d} t} \sum_{i=1}^{N_{+}}\left\langle\left[\mathbf{R}_{+, i}(t)-\mathbf{R}_{+, i}(0)\right]^{2}\right\rangle
$$

The sum term on the right-hand side of eq 10 over $N_{+}$is called the mean-square displacement (MSD). $N_{+}$is the number of diffusing counterions, $t$ is time, and $\mathbf{R}_{+, i}(t)$ is the position vector of a counterion $i$ at time event $t$. Average MSD curves as a function of time for each diffuser type are calculated. It is essential to notice that eq 10 is only valid when the Einstein diffusive regimen is reached. This means that the motion of the diffusing particle follows a random walk; in other words, its motion is not correlated with its motion at any previous time. To test the region in which eq 10 is valid, $\log (\mathrm{MSD})$ against $\log (t)$ was plotted in each case. The slope of the curve is $1.0 \pm 0.03,{ }^{13}$ when the Einstein diffusion is reached:

$$
\frac{\Delta \log \mathrm{MSD}}{\Delta \log t}=1
$$




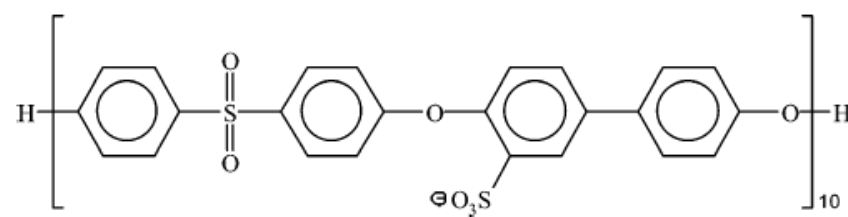

Figure 1. Scheme of a sulfonated poly(phenyl sulfone) chain with 10 repeating units.

Table 1. Description of the Different Simulated Cells

\begin{tabular}{ccrrccc}
\hline & \multicolumn{3}{c}{ number of particles } & $\begin{array}{c}\text { wt \% } \\
\text { cell water }\end{array}$ & $\begin{array}{c}\text { density } \\
\left(\mathrm{g} / \mathrm{cm}^{3}\right)\end{array}$ \\
\cline { 2 - 7 } & PPS10 & $\mathrm{H}_{2} \mathrm{O}$ & $\mathrm{H}^{+}$ & $\mathrm{H}_{3} \mathrm{O}^{+}$ & 10 & 1.32 \\
$\mathrm{~A}$ & 5 & 135 & 50 & 0 & 10 & 1.29 \\
$\mathrm{~B}$ & 5 & 240 & 50 & 0 & 18 & 1.26 \\
$\mathrm{C}$ & 5 & 400 & 50 & 0 & 30 & 1.32 \\
$\mathrm{D}$ & 5 & 85 & 0 & 50 & 10 & 1.29 \\
$\mathrm{E}$ & 5 & 190 & 0 & 50 & 18 & 1.26 \\
$\mathrm{~F}$ & 5 & 350 & 0 & 50 & 30 &
\end{tabular}

Computational Details. Bulk structures of poly(phenyl sulfone)s with sulfonic acid anions and water absorbed were generated and simulated by means of the Accelrys commercial software (Materials Studio 3.2) ${ }^{15}$ using the PCFF91 force field. ${ }^{16}$ All the MD simulation were performed under NVT conditions with the working temperature $T=300,320,340$, and $360 \mathrm{~K}$ controlled by means of the Andersen thermostat method with a collision ratio of 1.0. The density of the systems was assumed to be constant in the temperature range $300-360 \mathrm{~K}$, and consequently, the volume of each system was the same regardless of the working temperature. A time step $\delta=1 \mathrm{fs}$ (i.e., $10^{-15} \mathrm{~s}$ ) for the integration of the atomic motion equations was used. The van der Waals and Coulombic nonbonding interactions were calculated by the cell multipole method (CMM) $:{ }^{17-19}$ the value of the update width parameter was $1.0 \AA$, and the accuracy parameter was set to medium to use third order in the Taylor series expansion and explicit interactions form more neighboring cells. The structural results obtained by using the fast CMM methods for the cells agree with the results obtained with the slow Ewald summation method. ${ }^{20}$

Membranes consisting on 3D amorphous cells with periodic boundary conditions and containing different amounts of polymer, water and diffusion particles were built using the Amorphous_cell module of the software. Each cell contained five chains of 10 repeat units of poly(phenyl sulfone) (PES10) (Figure 1) and different amounts of $\mathrm{H}^{+}, \mathrm{H}_{3} \mathrm{O}^{+}$, and $\mathrm{H}_{2} \mathrm{O}$. The composition of the system contained in each cell is summarized in Table 1. Thus, cells A, B, and $\mathrm{C}$ contain naked protons whereas cells $\mathrm{D}, \mathrm{E}$, and $\mathrm{F}$ contain hydrated protons $\left(\mathrm{H}_{3} \mathrm{O}^{+}\right)$. The amount of water was $10 \mathrm{wt} \%$ in cells A and D, 18 wt \% in cells B and E, and 30 wt \% in cells C and $\mathrm{F}$.

The PCFF force field ${ }^{16,21-24}$ was developed to model polymers, and it has been found to be suitable for modeling PPS with sulfonic acids. ${ }^{25}$ Charges were calculated by the QEq charged 1.1 module which is available in Materials Studio software. The structure of each system was first minimized with respect to all the internal coordinates by a conjugate gradient method until the maximum derivative was smaller than $0.1 \mathrm{kcal} /(\AA \mathrm{mol})$, with a limit of 5000 steps. Then, the system was submitted to an equilibration process consisting on a $200 \mathrm{ps}\left(2 \times 10^{-10} \mathrm{~s}\right)$ long MD run. The data collecting stage consisted on MD runs of $2 \mathrm{~ns}\left(2 \times 10^{-9} \mathrm{~s}\right)$ for systems containing naked protons and $5 \mathrm{~ns}$ on the case of $\mathrm{H}_{3} \mathrm{O}^{+}$ hydrated protons. In both cases, the trajectories were saved each $500 \mathrm{fs}$ for subsequent analysis.

\section{Results and Discussion}

There is a vivid debate concerning both the nature of the water in wet ion-exchange membranes and the proton transport mechanism. The information at hand, ${ }^{27}$ corroborated by structural simulations of the membranes phase, suggests that water and protons are confined to domains of only nanometers, and proton conduction would be carried out in the domains through

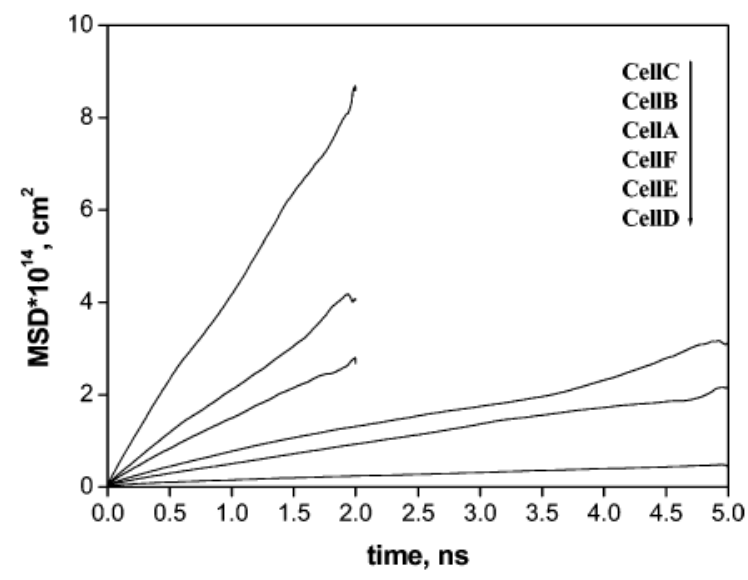

Figure 2. Mean-square displacement of $\mathrm{H}^{+}$(cells $\mathrm{A}, \mathrm{B}$, and $\mathrm{C}$ ) and $\mathrm{H}_{3} \mathrm{O}^{+}(\mathrm{D}, \mathrm{E}$, and $\mathrm{F})$ as a function of time at $300 \mathrm{~K}$.

a percolation path. Studies carried out by Paddison et al. ${ }^{27-30}$ on the dielectric spectrum of both Nafion and PEEK in the microwave region showed a strong dependence in the Nafion membranes with water content. This dependence is weaker for PEEK membranes owing to the stronger confinement of water in the narrow channels of these membranes. Quantification of the radial dependence of the dielectric permittivity of water in the nanopores of Nafion shows that as the water content increases the radius at which the dielectric permittivity starts to decrease diminishes. ${ }^{31,32}$ The confinement of the water in nanodimensional domains under a strong electrostatic field arising from the dissociated fixed ionic groups seems to keep the water molecules more tightly bound to each other and to the anionic fixed groups, thus inhibiting the structural diffusion of the protons. Moreover, they point out that hydrogen bonding between the sulfonic acid groups is favored, and it is likely that a minimal of water in the membrane forms a continuous path through which protons can diffuse. Finally, ab initio electronic structure calculations of polymer fragments with water and quantum molecular dynamics studies on model proton-exchange membranes suggest that the $\mathrm{Zundel}$ ion $\mathrm{H}_{5} \mathrm{O}_{2}{ }^{+}$may play an important role in the diffusion of protons in ion-exchange membranes with low water uptake. ${ }^{26}$

Theoretical models have been developed focused in the elucidation of ion transport in ion-exchange membranes. In these models, a certain amount of coarse-graining in the form of phenomenological parameters has been introduced. For example, Eikerling et al. ${ }^{32}$ developed a phenomenological model where the mobility of the protons is assumed to proceed via structure diffusion along the array of the anions of fixed groups over the interface and a bulk mechanism where the protons are transported with the Grotthus mechanism.

We have addressed proton transport in cation-exchange membranes using full dynamics considering the diffusion of naked protons and the hydrated $\mathrm{H}_{3} \mathrm{O}^{+}$particle. Possible formation of hydrophilic and hydrophobic domains is implicit in the conformational space landscape. Simulated protonic MSD values for cells A, B, and C, at four temperatures, are shown as a function of time in Figure 2. As expected, the values of MSD increase with time and temperature, the plots being straight lines of slopes close to unit in the whole time range. When the time is large enough, the diffusive regime is reached and $\left\langle\left[\mathbf{R}_{+}(t)-\right.\right.$ $\left.\left.\mathbf{R}_{+}(0)\right]^{2}\right\rangle$ varies linearly with time; i.e., the slope of the log$\left\langle\left[\mathbf{R}_{+}(t)-\mathbf{R}_{+}(0)\right]^{2}\right\rangle$ vs $\log (t)$ plot reaches unity, and the diffusion coefficient may be obtained from eq 10 . Values of the diffusion coefficient of single protons are plotted as a function of time in Figure 3. All the curves show a sharp decrease of the diffusion 


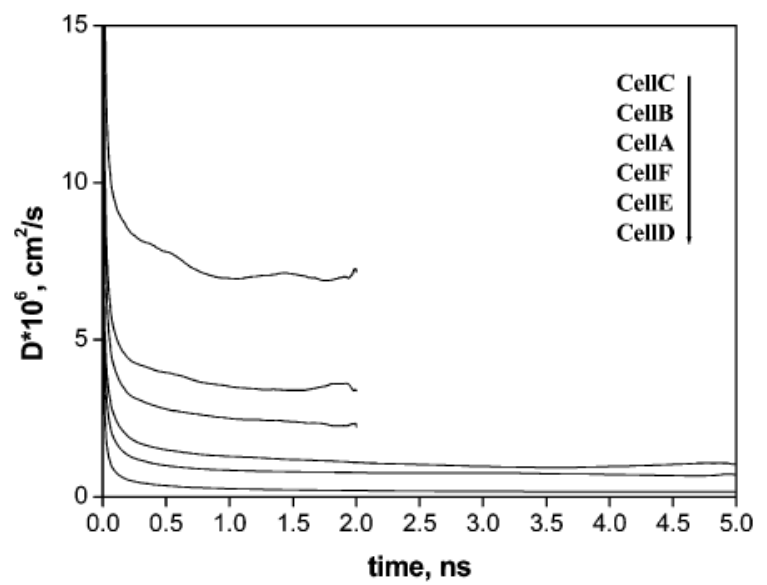

Figure 3. Evolution of the diffusion coefficient, in $\mathrm{cm}^{2} / \mathrm{s}$, with time for $\mathrm{H}^{+}$(cells $\mathrm{A}, \mathrm{B}$, and $\mathrm{C}$ ) and $\mathrm{H}_{3} \mathrm{O}^{+}$(cells $\mathrm{D}, \mathrm{E}$, and $\mathrm{F}$ ).

Table 2. Simulated Values of the Diffusion Coefficient, $D_{+}$, in $\mathrm{cm}^{2} / \mathrm{s}$, and the Electrical Conductivity, $\sigma$, in $\mathrm{S} / \mathrm{cm}$

\begin{tabular}{|c|c|c|c|c|c|c|c|c|}
\hline \multirow[b]{2}{*}{ cell } & \multicolumn{2}{|c|}{$300 \mathrm{~K}$} & \multicolumn{2}{|c|}{$320 \mathrm{~K}$} & \multicolumn{2}{|c|}{$340 \mathrm{~K}$} & \multicolumn{2}{|c|}{$360 \mathrm{~K}$} \\
\hline & $10^{6} D_{+}$ & $10^{3} \sigma$ & $10^{6} D_{+}$ & $10^{3} \sigma$ & $10^{6} D_{+}$ & $10^{3} \sigma$ & $10^{6} D_{+}$ & $10^{3} \sigma$ \\
\hline A & 2.32 & 21.4 & 3.05 & 26.4 & 4.79 & 39.1 & 4.92 & 37.9 \\
\hline B & 3.49 & 29.4 & 6.50 & 52.0 & 8.13 & 60.4 & 8.73 & 61.3 \\
\hline $\mathrm{C}$ & 6.99 & 52.5 & 9.90 & 69.7 & 14.19 & 94.0 & 16.83 & 104.4 \\
\hline $\mathrm{D}$ & 0.167 & 1.54 & 0.310 & 2.69 & 0.346 & 2.83 & 0.349 & 2.70 \\
\hline E & 0.720 & 6.08 & 1.01 & 7.98 & 1.160 & 8.65 & 1.45 & 10.2 \\
\hline F & 0.987 & 7.57 & 1.22 & 8.74 & 2.18 & 14.7 & 2.25 & 14.4 \\
\hline
\end{tabular}

coefficient with increasing time in the low times region, and the value of this quantity remains nearly constant at both moderate and long times. The values simulated for $D_{+}$at different temperatures for three cells with different water content are collected in the second, fourth, sixth, and eighth columns of Table 2. It can be seen that the diffusion coefficient of protons inside the membranes increases with both temperature and water content of the cells. Values of the conductivity calculated using the results simulated for $D_{+}$by eq 8 are given in the third, fifth, seventh, and ninth columns of Table 2 . The simulated conductivity is nearly 1 order of magnitude larger than the experimental result. The conductivity roughly follows Arrhenius behavior as the plots of Figure 4 show. A common characteristic of the results of Table 2 is that the protonic transport is associated with a low activation energy, which in the most unfavorable cases is lower than $3 \mathrm{kcal} \mathrm{mol}^{-1}$. The energy is higher than that predicted by Synder et al. based on the dynamic of bond percolation ${ }^{10}$ but lower than the experimental values reported for proton conduction in polyelectrolytes.

Let us consider now the full molecular dynamics for the diffusion of hydrated protons $\mathrm{H}_{3} \mathrm{O}^{+}$across membranes using the data of cells D, E, and F of Table 1. The trajectories for hydrated protons in Figure 2 are similar to those found for naked protons. Semilogarithmic plots of the $\mathrm{RMS}$ of $\mathrm{H}_{3} \mathrm{O}^{+}$shown as a function of time at different temperatures exhibit the same behavior as the naked protons in the sense that present a transitory process followed by steady-state conditions. The diffusion coefficient experiences a sharp drop with increasing time at short times, this coefficient remaining constant at moderate and long times (see Figure 3). As expected, the association of a proton with a molecule of water slows down the diffusion process of the hydrated particle, and the conductivity simulated for membranes with water uptake $18 \%$ and $30 \%$ is roughly similar to that experimentally found. Finally, the temperature dependence of the conductivity depicted in Figure 4 shows that this parameter for $\mathrm{H}_{3} \mathrm{O}^{+}$also follows Arrhenius behavior.

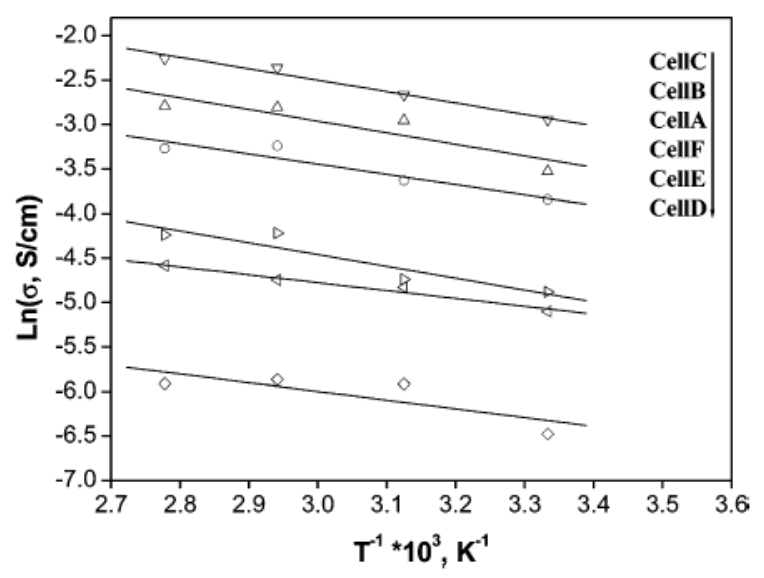

Figure 4. Arrhenius plots for the conductivity, in $\mathrm{S} / \mathrm{cm}$, of $\mathrm{H}^{+}$(cells $\mathrm{A}, \mathrm{B}$, and $\mathrm{C}$ ) and $\mathrm{H}_{3} \mathrm{O}^{+}$(cells $\mathrm{D}, \mathrm{E}$, and $\mathrm{F}$ ).

The distribution of the molecules of water in the conformational landscape of the polyelectrolyte was investigated by computing intermolecular pair correlations functions $g_{\mathrm{AB}}(r)$ which represent the probability of finding a pair of particles $\mathrm{AB}$ at a distance $r \pm \mathrm{d} r$ normalized with respect to the probability expected for a completely random distribution at the same density. ${ }^{34}$ Values of $g_{\mathrm{AB}}(r)$ were evaluated as ${ }^{15}$

$$
g_{\mathrm{AB}}(r)=\frac{V\left\langle\sum_{i \neq j} \delta\left(r-\left|r_{\mathrm{A} i}-r_{\mathrm{B} j}\right|\right)\right\rangle}{\left(N_{\mathrm{A}} N_{\mathrm{B}}-N_{\mathrm{AB}}\right) 4 \pi r^{2} \mathrm{~d} r}
$$

where $\mathrm{A}$ and $\mathrm{B}$ represent two kind of particles (i.e., individual atoms, ions such as $\mathrm{H}^{+}$, atomic groups like $\mathrm{H}_{3} \mathrm{O}^{+}$, etc.). The system has a volume $V$ and contains $N_{\mathrm{A}}$ particles of kind A and $N_{\mathrm{B}}$ particles of kind B with $N_{\mathrm{AB}}$ particles belonging simultaneously to both kinds ( $N_{\mathrm{A}}=N_{\mathrm{B}}=N_{\mathrm{AB}}$ when computing correlation among particles of the same kind, for instance, correlation of the relative positions of $\mathrm{H}^{+}$ions). Vectors $r_{\mathrm{A} i}$ and $r_{\mathrm{B} j}$ represent the position of particle $i$ of kind A and particle $j$ of kind $\mathrm{B}$, so that $\left|r_{\mathrm{A}}-r_{\mathrm{B} j}\right|$ is the distance between those two particles. The term $\delta\left(r-\left|r_{\mathrm{A} i}-r_{\mathrm{B} j}\right|\right)$ in eq 12 is set to unity when $\left(r-\left|r_{\mathrm{A} i}-r_{\mathrm{Bj} j}\right|\right) \leq \mathrm{d} r$ (i.e., when the difference between desired and actual distance among the two particles is smaller than a tolerance factor $\mathrm{d} r$ ) and to zero otherwise.

Values of $g(r)$ can be converted into coordination numbers with the following expression:

$$
n_{x \cdots z}(r)=4 \pi \frac{N_{z}}{\langle V\rangle} \int_{0}^{r} g_{x \cdots z}(s) s^{2} \mathrm{~d} s
$$

where $n_{x} \cdots_{z}(r)$ is the number of $x$ particles coordinated to particle $z$ within a radius $r,\langle V\rangle$ the cell volume, $N_{z}$ the total number of particles $z$ in the system, and $g_{x \cdots z}(s)$ the radial distribution function between $x$ and $z$. Pertinent results are collected in Table 3 . The main conclusion one can draw from the data of the table is that the coordination number for counterion particles $\left(\mathrm{H}^{+}\right.$or $\mathrm{H}_{3} \mathrm{O}^{+}$) around the sulfonic groups is slightly larger than unity. This means that the anion of the fixed group is compensated by the positive charge of the diffusant in such a way that the diffusive process may proceed by jumping of the counterion from a fixed group to a neighboring one. Accordingly, in the jumping step, some of the negative fixed groups anchored to the molecular chains could be compensated by their own proton plus the proton associated with other negative fixed group which is placed at a relatively short distance of the first one. This 
Table 3. Coordination Numbers of Fixed Groups and Mobile Particles

\begin{tabular}{|c|c|c|c|}
\hline$x \cdots z$ & system & $\begin{array}{l}\text { first coordination } \\
\text { shell }(\AA)\end{array}$ & $n(1)$ \\
\hline \multirow[t]{3}{*}{$\mathrm{S}$ in $\mathrm{SO}_{3}{ }^{-\cdots} \cdot \mathrm{H}^{+}$} & cell A & 3.37 & 1.4 \\
\hline & cell B & 3.55 & 1.2 \\
\hline & cell C & 3.37 & 1.2 \\
\hline \multirow[t]{3}{*}{$\mathrm{S}$ in $\mathrm{SO}_{3}{ }^{-} \cdots \mathrm{O}$ in $\mathrm{H}_{3} \mathrm{O}^{+}$} & cell D & 3.92 & 1.8 \\
\hline & cell E & 3.90 & 1.6 \\
\hline & cell F & 3.85 & 1.4 \\
\hline \multirow[t]{6}{*}{$\mathrm{S}$ in $\mathrm{SO}_{3}{ }^{-\cdots O}$ in $\mathrm{H}_{2} \mathrm{O}$} & cell A & 4.45 & 3.5 \\
\hline & cell B & 4.42 & 5.5 \\
\hline & cell C & 4.45 & 7.7 \\
\hline & cell D & 4.50 & 2.3 \\
\hline & cell E & 4.47 & 4.2 \\
\hline & cell F & 4.47 & 6.4 \\
\hline \multirow[t]{3}{*}{$\mathrm{H}^{+} \cdots \mathrm{O}$ in $\mathrm{H}_{2} \mathrm{O}$} & cell A & 2.65 & 1.5 \\
\hline & cell B & 2.65 & 2.5 \\
\hline & cell C & 2.65 & 3.5 \\
\hline \multirow[t]{3}{*}{$\mathrm{O}$ in $\mathrm{H}_{3} \mathrm{O}^{+} \cdots \mathrm{O}$ in $\mathrm{H}_{2} \mathrm{O}$} & cell D & 3.20 & 1.6 \\
\hline & cell E & 3.17 & 3.0 \\
\hline & cell F & 3.15 & 4.3 \\
\hline
\end{tabular}

mechanism would be responsible for the fact that each $-\mathrm{SO}_{3}{ }^{-}$ seems to be associated in the average with more than a single counterion.

\section{Conclusions}

The diffusion of naked protons in sulfonated phenyl polysulfones is nearly 1 order of magnitude larger than that of the hydrated protons. The value of the conductivity simulated for $\mathrm{H}_{3} \mathrm{O}^{+}$is roughly similar to that experimentally found. Simulated conductivities of hydrated protons follow Arrhenius behavior, but the activation energy is lower than that experimentally found for cation-exchange membranes. The coordination number between the fixed groups $\left(-\mathrm{SO}_{3}{ }^{-}\right)$and the counterion particles $\left(\mathrm{H}^{+}\right.$or $\left.\mathrm{H}_{3} \mathrm{O}^{+}\right)$is slightly larger than unity. Although the rather good agreement between simulated and experimental conductivities is stimulating, more work must be carried out in polyelectrolytes with different chemical structures to assess the reliability of full molecular dynamics to predict proton conductivities of cation-exchange membranes as a function of the chemical structure.

Acknowledgment. This work was supported by the Comunidad de Madrid through the grant interfaces S-0505/MAT-0227, Fondos Europeos de Desarrollo (FEDER), and Fondo Social Europeo (FSE). Financial support was also supplied by the Dirección General de Investigación Científica y Técnica (DGICYT) (grant MAT 2005-05648-C02-02).

\section{References and Notes}

(1) Wyllie, J. R.; Patnode, H. W. J. Phys. Chem. 1950, 54, 204.

(2) Juda, W.; McRae, W. A. J. Am. Chem. Soc. 1950, 72, 1044.

(3) Wilson, J. Demineralization by Electrodyalisis; Butterworths: London, 1960.

(4) Lakshminarayanaiah, N. Transport Phenomena in Membranes; Academic Press: London, 1967.

(5) Riande, E. In Physics of Electrolytes; Academic Press: New York, 1972; Vol. 1, Chapter 11.

(6) For a review: Hickner, M. A.; Ghassemi, H.; Kim, Y. S.; Einsla, B. R.; McGrath, J. E. Chem. Rev. 2004, 104, 4587.

(7) Neyertz, S.; Brown, D. J. Chem. Phys. 1996, 104, 3797.

(8) Müller-Plathe, F.; van Gunsteren, W. F. J. Chem. Phys. 1995, 103, 4745.

(9) Leeuw, S. W; van Zon, A.; Bel, G. J. Electrochim. Acta 2001, 46, 1419.

(10) Synder, J. F.; Ratner, M. A.; Shriver, D. F. Solid State Ionics 2002, $147,249$.

(11) Paddison, S. J. Annu. Rev. Mater. Res. 2003, 33, 289.

(12) Parcero Herrera, E. Estudio de la fosfonación y sulfonación de polisulfonas para producir membranas para celdas de combustible de metanol directo. Master Thesis, Universidad Nacional Autónoma de México (UNAM), 2004.

(13) Müller-Plathé F. Acta Polym. 1994, 45, 259.

(14) Lonergan, M. C. Ion Transport in Polymer Electrolytes. Ph.D. Thesis, Northwestern University, Evanston, IL, 1994; p 319.

(15) Accelrys Inc, San Diego, CA (Materials Studio 3.2, VISUALIZER, AMORPHOUS CELL and DISCOVER modules).

(16) Sun, H.; Mumby, S. J.; Maple, J. R.; Hagler, A. T. J. Am. Chem. Soc. $1994,116,2978$.

(17) Greengard, L; Rokhlin, V. I. J. Comput. Phys. 1987, 73, 325.

(18) Schmidt, K. E.; Lee, M. A. J. Stat. Phys. 1991, 63, 1223.

(19) Ding, H. Q.; Karasawa, N.; Goddard, W. A. J. Chem. Phys. 1992, 97, 4309.

(20) Ewald, P. P. Ann. Phys. 1921, 64, 253.

(21) Sun, H. J. Comput. Chem. 1994, 15, 752.

(22) Sun, H. Macromolecules 1995, 28, 701.

(23) Hill, J.-R; Sauer, J. J. Phys. Chem. 1994, 98, 1238.

(24) Maple, J. A.; Hwang, M. J.; Stockfisch, T. P.; Dinur, U.; Waldman, M.; Ewig, C. S.; Hagler, A. T. J. Comput. Chem. 1994, 15, 162.

(25) Ennari, J.; Hamara, J.; Sundholm, F. Polymer 1997, 38, 3733.

(26) For a review: Paddison, S. J. Annu. Rev. Mater. Res. 2003, 33, 289.

(27) Paddison, S. J.; Reagor, D. W.; Zawodzinski, T. A., Jr. J. Electroanal. Chem. 1998, 459, 91.

(28) Paddison, S. J.; Bender, G.; Kreuer, K. D.; Nicoloso, N.; Zawodzinski, T. A., Jr. J. New Mater. Electrochem. Syst. 2000, 3, 291.

(29) Paddison, S. J.; Pratt, L. R. Zawodzinski, T. A., Jr. J. Phys. Chem. A 2001, 105, 6266.

(30) Eikerling, M.; Paddison, S. J.; Pratt, L. R.; Zawodzinski, T. A., Jr. Chem. Phys. Lett. 2003, 368, 108.

(31) Paul, R; Paddison, S. J. J. Chem. Phys. 2001, 115, 7762.

(32) Paul, R.; Paddison, S. J. Solid State Ionics 2004, 168, 245.

(33) Eikerling, M.; Kornyshev, A. A.; Stimming, U. J. Phys. Chem. B 1997, 101, 10807.

(34) Allen, M. P.; Tildesley, D. J. Computer Simulation of Liquids; Clarendon Press: Oxford, UK, 1987.

MA062070H 